${ }^{1}$ Departamento de Medicina Interna. Facultad de Medicina. Universidad de Chile. Santiago, Chile. Unidad de Paciente Crítico. Hospital Clínico Universidad de Chile. Santiago, Chile.

${ }^{3}$ Escuela de Medicina. Universidad de Chile. Santiago, Chile. ${ }_{4}^{4}$ Departamento de Terapia Ocupacional y Ciencia de la Ocupación. Universidad de Chile. Santiago, Chile.

${ }^{5}$ Escuela de Terapia Ocupacional, Facultad Ciencias de la Salud. Universidad Central de Chile. Santiago, Chile.

${ }^{6}$ Departamento Medicina Intensiva. Pontificia Universidad Católica de Chile. Santiago, Chile.

${ }^{7}$ Dirección de Postítulo. Escuela de Medicina. Universidad Finis Terrae. Santiago, Chile. ${ }^{8}$ Unidad de Cuidados Intensivos. Hospital San Juan de Dios. Santiago, Chile. ${ }^{9}$ Proyecto Internacional de Investigación para la Humanización de los Cuidados

Intensivos. Proyecto HUCl, España.

${ }^{10}$ Medicina Física y Rehabilitación. Hospital Clínico Universidad de Chile. Santiago, Chile.

${ }^{11}$ Centro de Investigación Clínica Avanzada (CICA). Hospital Clínico Universidad de Chile. Santiago, Chile. a'Enfermera. ${ }^{b}$ Interno de Medicina. 'Terapeuta Ocupacional. ${ }^{\mathrm{d}}$ Farmacéutico Clínico.

Trabajo no recibió financiamiento. Los autores declaran no tener conflictos de interés.

Recibido el 17 de enero de 2020, aceptado el 28 de abril de 2021 .

Correspondencia a: Eduardo Tobar Almonacid Carlos Lorca \#999, Santiago. etobar@hcuch.cl

\section{Prácticas nacionales de analgesia, sedación y delirium en las Unidades de Cuidados Intensivos de adultos en Chile}

\author{
VERÓNICA ROJAS $S^{1,9,11, a}$, CARLOS ROMERO ${ }^{1,2}$, \\ DANIEL TOBAR ${ }^{3, \mathrm{~b}}$, EVELYN ALVAREZ $^{4,5, \mathrm{c},}$, \\ ROLANDO ARANDA ${ }^{1,10, \mathrm{c}}$, GUILLERMO BUGEDO ${ }^{6}$, \\ MARCIAL CARIQUEO ${ }^{1,2, \mathrm{~d}}, \mathrm{M}$. IDALIA SEPÚLVEDA ${ }^{7, \mathrm{a}}$, \\ JUAN EDUARDO SÁNCHEZ ${ }^{8}$, EDUARDO TOBAR ${ }^{1,2}$
}

\section{Analgesia, sedation, neuromuscular blockade and delirium management practices in Chilean intensive care units}

Background: The appropriate use of analgesia, sedation, neuromuscular blockade and the diagnosis and prevention of delirium (ASBD) are associated with better outcomes in critically ill patients at Intensive Care Unit (ICUs). Aim: To know the practices about analgesia, sedation, delirium, and neuromuscular blockade use among healthcare professionals working in adult ICUs in Chile. Material and Method: An electronic survey was sent to 812 professionals working in ICUs using a previously published instrument, which was adapted and authorized by the author. Results: We received 278 surveys. Fifty two percent of respondents were physicians, 34\% nurses and 11\% physical therapists. Their age ranged between 30 and 39 years in $43 \%$ and was over 50 years in 9\%. Eighty four percent evaluated pain routinely, but only $26 \%$ use a validated scale. Sedation was routinely evaluated with a validated scale and $73 \%$ referred to have a protocol. Neuromuscular block is seldom used, and little monitoring occurs (43\%). Delirium is routinely evaluated by $48 \%$ of respondents, usually using the CAM-ICU scale. Conclusions: There is a heterogeneous adherence to the $A S B D$ recommended practices. The main gaps are in the assessment of pain, monitoring of neuromuscular blockade and diagnosis of delirium through validated instruments.

(Rev Med Chile 2021; 149: 864-872)

Key words: Critical Care; Delirium; Hypnotics and Sedatives; Neuromuscular Blockade.
$\mathrm{E}$ 1 uso adecuado de analgesia, sedación y bloqueadores neuromusculares (BNM) ha sido asociado a mejores resultados en los pacientes críticos ${ }^{1-4}$. El delirium ha sido reconocido como una condición que impacta en el pronóstico cognitivo y funcional en el mediano y largo plazo ${ }^{5,6}$. Los últimos años, se ha generado evidencia que demuestra que mediante estrategias farmacológicas y no farmacológicas es posible reducir los riesgos y complicaciones asociadas a la aparición del delirium, así como los efectos adversos derivados del uso inapropiado 
de analgesia, sedación y $\mathrm{BNM}^{7-12}$; sin embargo, reportes internacionales muestran que la implementación de esta evidencia a nivel global aún es insuficiente $\mathrm{e}^{13-16}$.

La reciente publicación realizada por la Sociedad Americana de Cuidados Críticos de las Guías de práctica clínica para el manejo del dolor, sedación/agitación, delirium, inmovilización y disrupción del sueño, en pacientes críticos ${ }^{17,18}$, sugieren la realización de estudios locales orientados a conocer las prácticas y factores que representen barreras y/o facilitadores en el proceso de implementación, así como las percepciones y creencias de los profesionales de la salud en relación a la temática ${ }^{19}$.

En virtud de estas recomendaciones internacionales, y como parte de un proyecto liderado por la Sociedad Chilena de Medicina Intensiva (SOCHIMI) para generar recomendaciones nacionales basadas en la evidencia y considerando la realidad local, se diseñó un estudio observacional con el objetivo de conocer las prácticas nacionales del equipo de salud en relación a esta temática.

\section{Material y Método}

Se realizó un estudio descriptivo, no intervencional, mediante una encuesta anónima y voluntaria aplicada vía electrónica a profesionales de la salud que trabajan en UCI de adultos en Chile. Con la autorización del autor principal, se realizó la traducción y adaptación de un instrumento previamente publicado por un grupo latinoamericano ${ }^{19}$. El instrumento consta de 5 secciones: La sección 1 explora datos sociodemográficos de la institución y el profesional que responde la encuesta. La sección 2 evalúa si existe medición rutinaria de la presencia de dolor y los instrumentos diagnósticos utilizados. Las preguntas respecto a sedación están contenidas en la sección 3, evaluándose el uso de escalas, existencia de protocolos, suspensión de infusiones, y fármacos empleados. En la sección 4, se evalúa el uso de BNM y su monitoreo. Finalmente, la sección 5 evalúa conocimientos y percepciones acerca del delirium a través de escala tipo Likert, las opciones fueron totalmente de acuerdo, de acuerdo, indiferente, en desacuerdo, y totalmente en desacuerdo. Además se evaluó la implementación de medidas de prevención no farmacológica (PNFD) y manejo farmacológico del delirium.

Una vez efectuada la traducción del instrumento, se llevó a cabo una prueba piloto de la versión inicial en 10 profesionales de la salud, realizándose cambios menores a partir de las sugerencias recogidas. De ese proceso surge la versión final de la encuesta, que fue aplicada del 01 agosto hasta el 30 de septiembre de 2017, mediante formulario electrónico Google Forms enviado por correo electrónico a los socios de la SOCHIMI. Además, se habilitó en la página web de la sociedad (www. medicina-intensiva.cl) un acceso directo para responderla, se enviaron correos electrónicos recordatorios y se difundió en las actividades académicas de la SOCHIMI.

El objetivo primario del estudio fue conocer las prácticas relacionadas a la analgesia, sedación, delirium y uso de BNM (ASBD) de profesionales de la salud que trabajan en UCI de adultos en Chile. Como objetivo secundario, el estudio exploró si las características de los profesionales encuestados, se asociaban a las prácticas en este tópico.

Para el análisis de los datos descriptivos, se consideraron sólo las encuestas completas, expresándose los resultados mediante proporciones (\%). Para la comparación de características de los encuestados, y las percepciones y prácticas evaluadas, se realizaron pruebas de chi cuadrado con un valor de significación $p<0,05$. Para el análisis de las preguntas de conocimientos y percepciones en relación al delirium, se agruparon las 5 categorías originales en 3: de acuerdo, indiferente, en desacuerdo. Se utilizó el programa estadístico Prism 7 (GraphPad INC).

El presente estudio fue aprobado por el Comité de Ética de Investigación Científica del Hospital Clínico de la Universidad de Chile. Al momento de responder la encuesta, los participantes dieron su consentimiento para el uso de la información recolectada como material de apoyo en la realización de guías nacionales, así como para su posterior publicación.

\section{Resultados}

Se invitó a responder la encuesta a 812 profesionales y se recibieron 278 encuestas completas (34\%). Las características de los individuos que respondieron el instrumento, así como de las 
UCI en las cuales se desempeñan, se exponen en la Tabla 1.

Respecto a las prácticas de analgesia, $84 \%$ de los encuestados señala que en su unidad se evalúa la presencia de dolor de forma rutinaria, siendo la frecuencia de evaluación: $21 \%$ una vez por turno, $30 \%$ dos veces por turno y $49 \%$ tres o más veces por turno. Respecto a la escala empleada, $46 \%$ utiliza la escala visual análoga (EVA), 14\% la escala conductual del dolor (ECD) y $11 \%$ la Critical Pain Observational Tool (CPOT). Un 10\% emplea de forma aislada los signos vitales. Las prácticas de sedación y uso de BNM se pueden observar en la Tabla 2.

En relación al delirium, las prácticas de monitorización y percepción de su prevalencia se exponen en la Tabla 3. Respecto al nivel de conocimiento y percepción acerca del delirium, los resultados se pueden apreciar en la Figura 1. En la Figura 2 se muestra la percepción del nivel de implementación de las estrategias de PNFD y en la Figura 3 es posible identificar las barreras para la implementación de estas. Los fármacos más empleados para el manejo del delirium fueron antipsicóticos atípicos $(92 \% ; \mathrm{n}=257)$, dexmedetomidina $(60 \% ; \mathrm{n}=167)$ y haloperidol $(53 \%$; $\mathrm{n}=148)$.

Respecto a las estrategias para mejorar las prácticas de (ASBD) 66\% de los participantes señaló que en su lugar de trabajo se realiza una visita diaria interprofesional y los miembros del equipo de salud que participan mayoritariamente son: $95 \%$ enfermeras, $95 \%$ médicos, $86 \%$ kinesiólogos, 54\% nutricionistas y 52\% técnicos paramédicos. El $87 \%$ señala que la existencia de un protocolo escrito favorecería el cumplimiento de las prácticas, $87 \%$ refiere que se deben entrenar enfermeros y $62 \%$ cree que la presencia de un Químico Farmacéutico en las rondas clínicas sería beneficiosa.

Finalmente, en relación a la exploración de la asociación entre las características de los profesionales y sus percepciones, se encontró que los médicos expresan una mayor percepción de la aplicación de protocolos de sedación $(\mathrm{p}=0,01)$ y de la evaluación de delirium $(\mathrm{p}<0,01)$, respecto a los otros profesionales de la salud. En relación al nivel de confianza para identificar el delirium, este es mayor entre los médicos $(p=0,01)$ y en los profesionales con más de 5 años de desempeño laboral $(\mathrm{p}=0,03)$.
Tabla 1. Características sociodemográficas, y del lugar de trabajo de los profesionales de la salud encuestados ( $n: 278)$

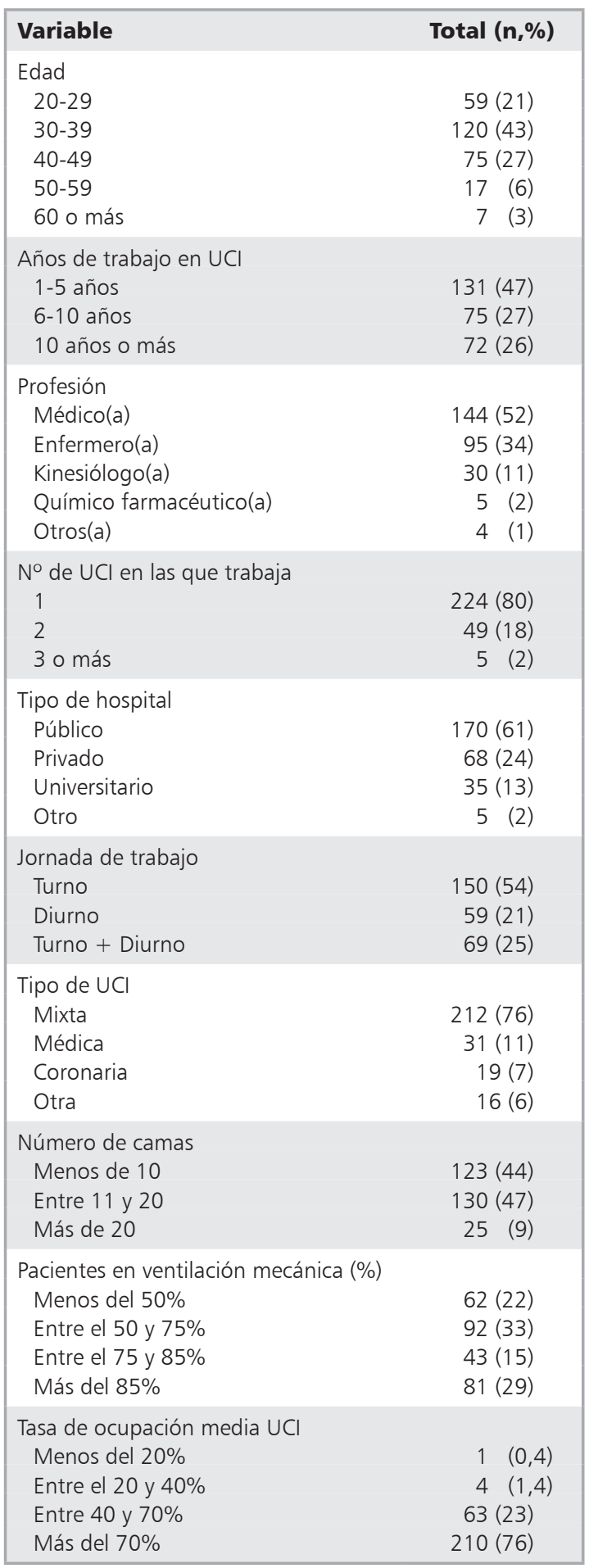


Tabla 2. Prácticas de Sedación y Bloqueo Neuromuscular en las UCI de Chile (n: 278)

\begin{tabular}{|c|c|}
\hline Variable & Total (n, \%) \\
\hline $\begin{array}{l}\text { Existe protocolo de sedación } \\
\text { Sí } \\
\text { No }\end{array}$ & $\begin{array}{r}\mathrm{n}: 278 \\
202(73) \\
76(27)\end{array}$ \\
\hline $\begin{array}{l}\text { Adherencia al protocolo } \\
\text { Nunca } \\
\text { Menos del } 25 \% \\
\text { Entre el } 25 \text { y } 50 \% \\
\text { Entre el } 50 \text { y } 75 \% \\
\text { Más del } 75 \%\end{array}$ & $\begin{array}{r}\mathrm{n}: 202 \\
3(1,5) \\
15(7,4) \\
27(13,4) \\
45(22,2) \\
112(55,5)\end{array}$ \\
\hline $\begin{array}{l}\text { Discusión de metas diarias } \\
\text { Nunca } \\
\text { Menos del } 25 \% \\
\text { Entre el } 25 \text { y } 50 \% \\
\text { Entre el } 50 \text { y } 75 \% \\
\text { Más del } 75 \%\end{array}$ & $\begin{array}{l}\mathrm{n}: 278 \\
14 \quad(5) \\
38(14) \\
50(18) \\
55(20) \\
121(43)\end{array}$ \\
\hline $\begin{array}{l}\text { Utiliza escala de evaluación } \\
\text { Sí } \\
\text { No }\end{array}$ & $\begin{array}{r}\mathrm{n}: 278 \\
270(97) \\
8 \quad(3)\end{array}$ \\
\hline $\begin{array}{l}\text { Frecuencia de evaluación } \\
1 \text { vez por día } \\
2 \text { veces por día } \\
3 \text { veces por día } \\
\text { Más de } 3 \text { veces }\end{array}$ & $\begin{array}{c}\mathrm{n}: 270 \\
19 \quad(7) \\
62(23) \\
28(10) \\
161(60)\end{array}$ \\
\hline $\begin{array}{l}\text { Escala usada } \\
\text { Ramsay } \\
\text { SAS } \\
\text { RASS } \\
\text { Otra }\end{array}$ & $\begin{array}{c}\text { n: } 270 \\
9 \quad(3) \\
220(82) \\
38(14) \\
3 \quad(1)\end{array}$ \\
\hline $\begin{array}{l}\text { Interrupción diaria de sedantes } \\
\text { No } \\
\text { Sí }\end{array}$ & $\begin{array}{r}\mathrm{n}: 278 \\
191(69) \\
87(31)\end{array}$ \\
\hline $\begin{array}{l}\text { Frecuencia de interrupción } \\
\text { Menos del } 25 \% \\
\text { Entre el } 25 \text { y } 50 \% \\
\text { Entre el } 50 \text { y } 75 \% \\
\text { Más del } 75 \%\end{array}$ & $\begin{array}{c}\mathrm{n}: 87 \\
20(23) \\
29(33) \\
21(24) \\
17(20)\end{array}$ \\
\hline $\begin{array}{l}\text { Frecuencia de BNM } \\
\text { Menos del } 25 \% \\
\text { Entre el } 25 \text { y } 50 \% \\
\text { Entre el } 50 \text { y } 75 \% \\
\text { Más del } 75 \% \\
\text { Nunca }\end{array}$ & 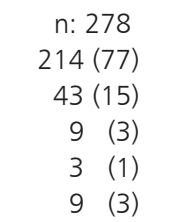 \\
\hline $\begin{array}{l}\text { Monitorización BNM } \\
\text { Escala de sedación } \\
\text { Train of Four (TOF) } \\
\text { Indice Biespectral (BIS) } \\
\text { BIS+TOF } \\
\text { Nada }\end{array}$ & $\begin{array}{l}\mathrm{n}: 269 \\
57(21) \\
66(24) \\
23(9) \\
52(19) \\
71(26)\end{array}$ \\
\hline
\end{tabular}

Tabla 3. Prácticas nacionales de monitorización, y percepciones en relación al delirium en la UCI (n: 278)

\begin{tabular}{|lc|}
\hline Variable & Total (n, \%) \\
\hline Evaluación rutinaria del delirium & $\mathrm{n}: 278$ \\
Sí & $132(48)$ \\
No & $146(52)$ \\
Frecuencia de evaluación & $\mathrm{n}: 132$ \\
1 vez al día & $34(26)$ \\
2 veces al día & $44(33)$ \\
3 veces o más & $15(11)$ \\
Nivel de confianza para identificar delirium & $\mathrm{n}: 278$ \\
Mucha confianza & $54(19)$ \\
Moderada confianza & $154(55)$ \\
Algo de confianza & $50(18)$ \\
Escasa de confianza & $14(5)$ \\
No estoy seguro & $2(1)$ \\
Ninguna & $4(2)$ \\
Escala usada para evaluar delirium & $\mathrm{n}: 132$ \\
CAM-ICU & $92(70)$ \\
Evaluación Clínica & $38(29)$ \\
Otra & $2(1)$ \\
\hline
\end{tabular}

\section{Discusión}

El presente trabajo corresponde al primer estudio que, mediante una encuesta a profesionales de la salud que trabajan en UCI, recoge información sobre los conocimientos, percepciones y creencias respecto a las prácticas de ASBD en las UCI de adultos a nivel nacional. Previamente, integrantes de nuestro equipo, realizaron un estudio multicéntrico nacional que describió el uso de analgesia, sedación y BNM, pero fue llevado a cabo hace más de 10 años y no incluyó delirium ${ }^{20}$. Nuestros hallazgos documentan una heterogénea adherencia a las recomendaciones internacionales para el manejo de la ASBD en los pacientes críticos sometidos a ventilación mecánica invasiva (VMI). Entre los hallazgos centrales a destacar se encuentran:

1) Una elevada proporción de los encuestados refiere que en su unidad se evalúa rutinariamente el dolor; sin embargo, el uso de escalas validadas en pacientes en VMI es baja $(25 \%)$. 


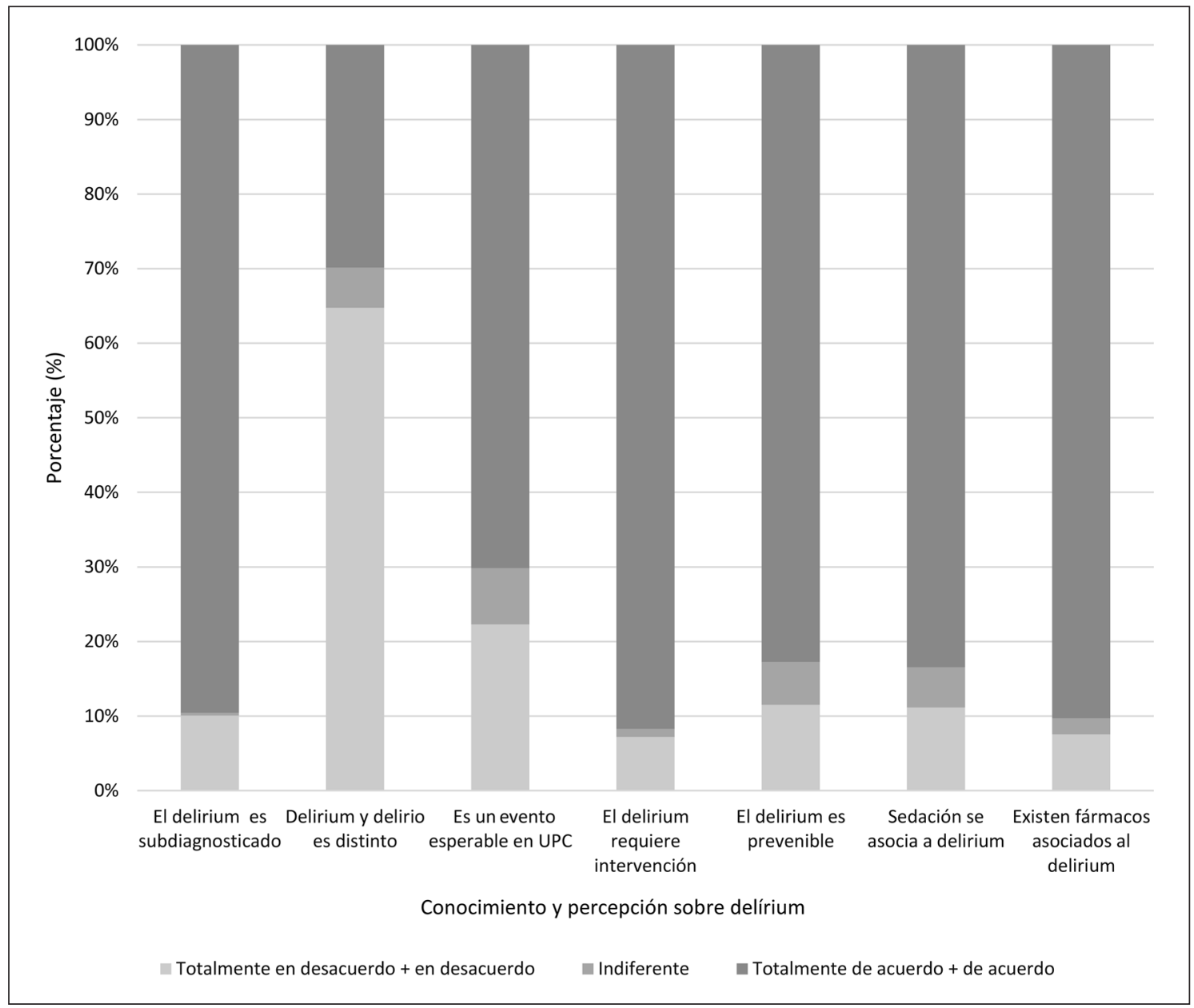

Figura 1. Conocimiento y percepción sobre delirium de profesionales de salud que trabajan en UCl adulto en Chile ( $\mathrm{n}$ : 278).

2) Existe un adecuado reporte del uso de escalas validadas para la evaluación de la sedación y el empleo de protocolos de analgesia-sedación, con la percepción de una elevada adherencia.

3) El uso de BNM reportado es bajo, lo que sería concordante con las recomendaciones internacionales vigentes; pero el uso de sistemas de monitorización es bajo.

4) Existe una elevada percepción de que el delirium es subdiagnosticado (90\%) y que es una condición prevenible (83\%). No obstante, existe un escaso manejo de su definición y un bajo empleo de escalas validadas para su diagnóstico.
5) La percepción de implementación de estrategias de PNFD es parcial y en relación al manejo farmacológico del delirium es frecuente el uso de antipsicóticos atípicos.

6) En relación a las barreras para mejorar el manejo del delirium destacan la poca conciencia de su importancia, el escaso conocimiento e insuficiente coordinación de los equipos interprofesionales de cuidados críticos.

7) Los equipos médicos tienen una mayor percepción de la implementación de los instrumentos de evaluación y protocolos, que los otros profesionales de la salud.

A continuación, revisaremos en mayor pro- 


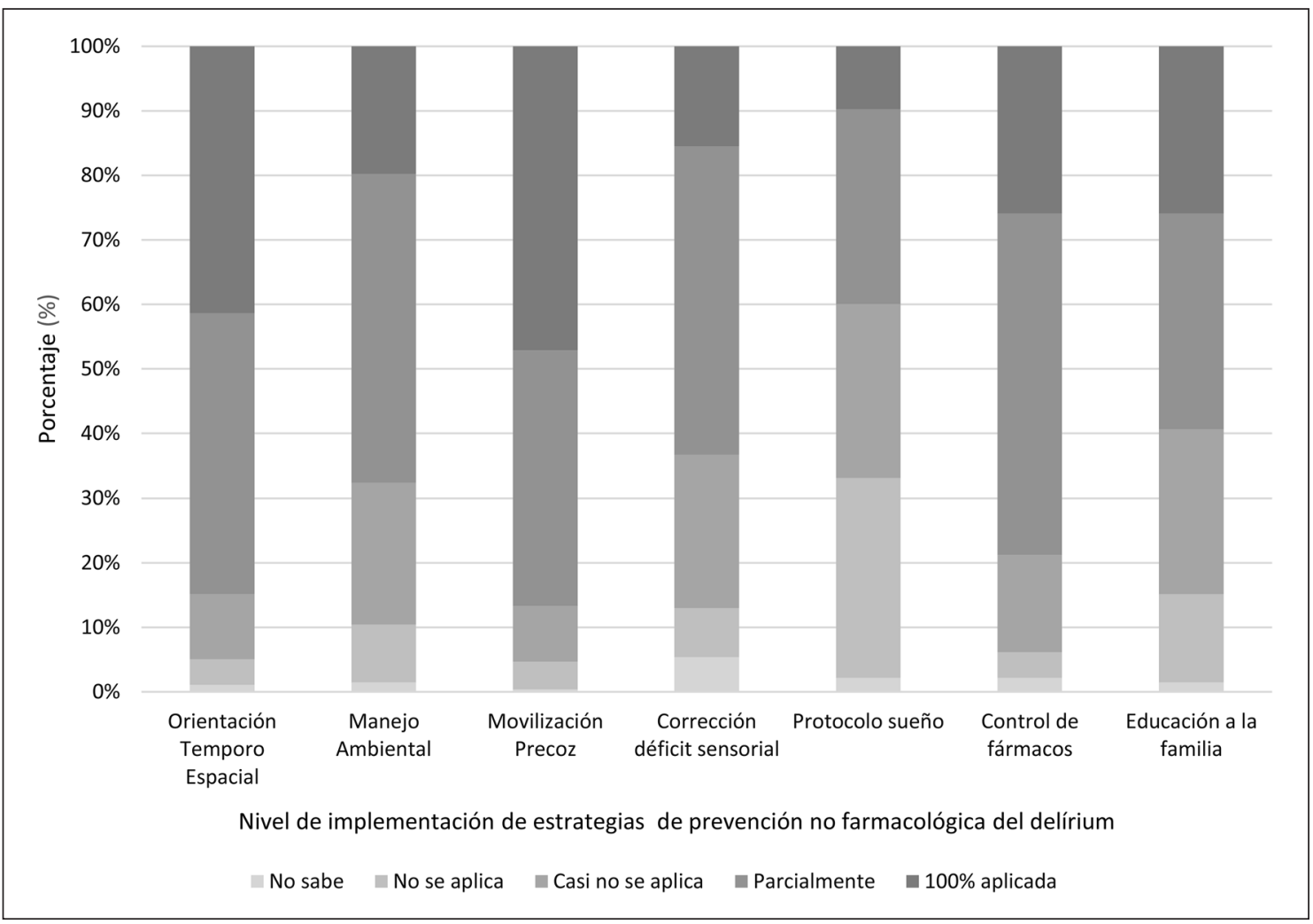

Figura 2. Percepción de profesionales de salud que trabajan en UCl adulto de Chile sobre el nivel de implementación de estrategias de prevención no farmacológica del delirium (n: 278).

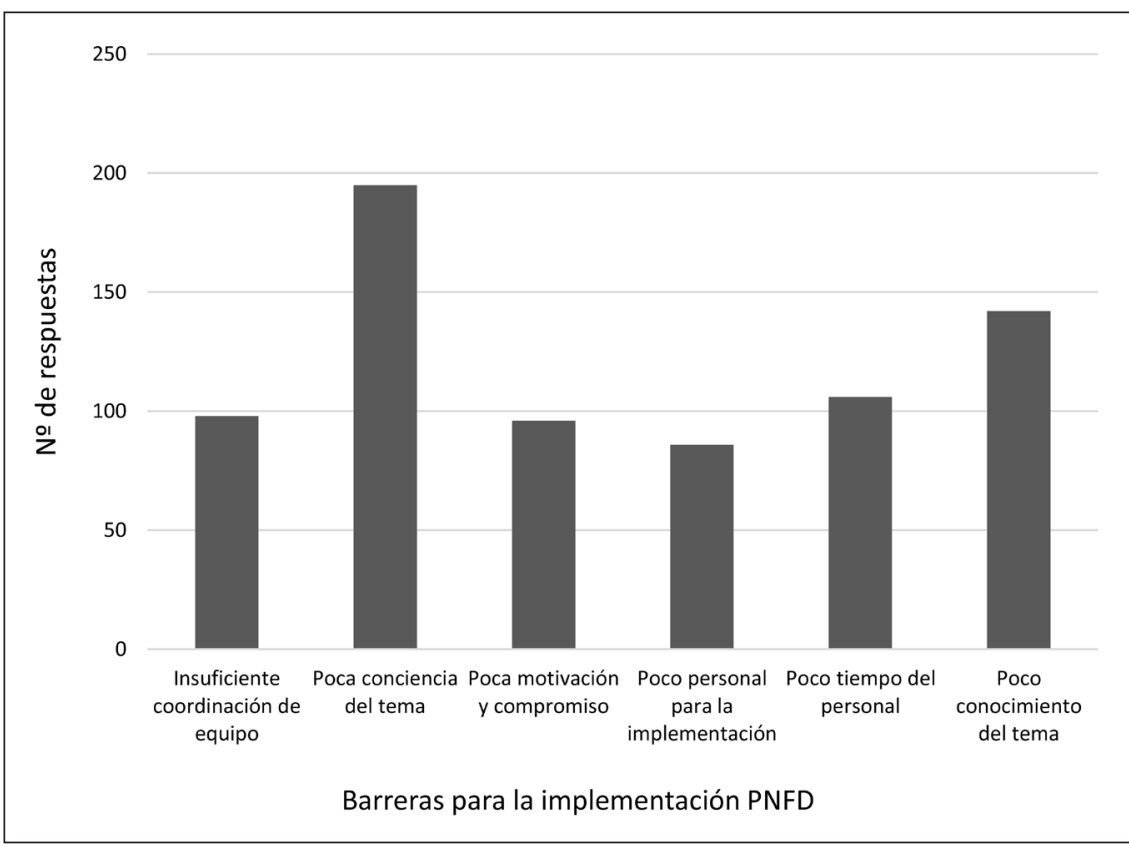

Figura 3. Barreras para la implementación de estrategias de prevención no farmacológica del delirium (n: 278). 
fundidad los resultados encontrados, comparando nuestros hallazgos con la información nacional y global cuando corresponda.

En relación a analgesia, pese a declararse un alto nivel de monitoreo del dolor, la aplicación de escalas validadas como son ECD o CPOT ocurre solo en $14 \%$ y $11 \%$ respectivamente, siendo EVA (46\%) la escala más utilizada. Esta realidad coincide con las prácticas internacionales reportadas en la encuesta mundial del bundle ABCDEF, en que el uso de EVA alcanza 54\%, ECD 27\% y CPOT $17 \%{ }^{21}$. Un reporte local, realizado en UCI de la Región Metropolitana el año 2015, evidenció que sólo $3 \%$ evaluaba dolor con una escala validada ${ }^{22}$.

Por otra parte, el uso de instrumentos validados y la existencia de protocolos de sedación mostraron niveles de adherencia adecuados (78\%), lo que estuvo en concordancia con lo encontrado en la encuesta mundial ABCDEF ${ }^{21}$. RASS (Richmond Agitation-Sedation Scale) es la más usada a nivel global, en tanto a nivel nacional predomina el uso de SAS (Sedation-Agitation Scale), que ha sido empleado en los estudio nacionales ${ }^{20,21,23}$. El uso de la estrategia de suspensión diaria de la infusión de sedantes es baja, 69\% de los encuestados señala no realizarla. Un metaanálisis sobre esta práctica, sugiere que es más frecuente su uso en países desarrollados ${ }^{24}$. Es importante considerar que en muchas UCI de países desarrollados la relación enfermera:paciente es de 1:1. Mehta y cols. ${ }^{25}$, demostraron que en pacientes adultos sometidos a VMI y manejados con un esquema de sedación protocolizada, la incorporación de interrupción diaria de la infusión de sedantes no redujo la duración de la VMI, ni la estadía en la UCI. Nuestros hallazgos sugieren que a nivel nacional predomina el uso de protocolos locales de analgesia y sedación sobre la estrategia de suspensión diaria.

Acerca del BNM, 77\% de los encuestados señaló que la frecuencia de uso es menor a $25 \%$; sin embargo, el uso de sistemas de monitoreo recomendados (TOF y TOF+BIS) fue inferior a $43 \%$, similar a lo observado por otros investigadores ${ }^{13,26}$.

Pese a reconocerse el delirium como un fenómeno de importancia, menos de la mitad de los encuestados refiere evaluarlo en forma rutinaria. La escala CAM-ICU (Confusion Assessment Method for the Intensive Care Unit) es la más empleada a nivel nacional,en acuerdo con lo publicado a nivel global por Morandi y cols. ${ }^{21}$. En cuanto al manejo del delirium, los profesionales señalaron que el uso de fármacos es habitual, siendo los más frecuentes: antipsicóticos atípicos, dexmedetomidina y haloperidol. Nuestros hallazgos contrastan con las recomendaciones vigentes, en las cuales ante la falta de evidencia que sustente el beneficio del uso de neurolépticos en el manejo del delirium, se sugieren sólo de manera restringida en casos con agitación psicomotora moderada a severa sin respuesta a manejo no farmacológico ${ }^{17,27,28}$.

Sobre el nivel de conocimiento y percepciones en relación al delirium, algunos de nuestros resultados coinciden con otras series de la región. Salluh y cols. ${ }^{19}$, en un estudio efectuado en Brasil encontraron que $84 \%$ creía que el delirium era subdiagnosticado y $82 \%$ que su desarrollo se asociaba al uso sedantes. Importante de señalar es que, en nuestro estudio, una elevada proporción de los encuestados considera que el delirium es prevenible $(83 \%)$, situación que difiere de lo reportado por otros autores: $67 \%$ y $20 \%$ respectivamente ${ }^{19,29}$. Las estrategias de PNFD en las que se percibe un elevado nivel de cumplimiento son: orientación temporoespacial y movilización precoz; parcial nivel de cumplimiento en: control de fármacos, manejo ambiental y corrección de déficit sensorial, y bajo nivel de cumplimiento en: disponibilidad de protocolos de sueño y educación a la familia. Estos hallazgos, coinciden con lo reportado globalmente ${ }^{21}$.

En nuestros encuestados el nivel de conocimiento, conciencia del tema y tiempo del personal son percibidas como las principales barreras para la implementación de PNFD. Xiang y cols. ${ }^{15}$, han publicado resultados similares en una encuesta aplicada a profesionales que trabajan en UCI en China.

La mayor percepción del equipo médico respecto a su nivel de confianza para evaluar delirium y el cumplimiento de protocolos por sobre los otros profesionales de salud coincide con lo publicado en Europa y Asia ${ }^{14,29}$. La asociación entre años de práctica profesional y el nivel de confianza para evaluar delirium, también ha sido descrita por otros investigadores ${ }^{30}$; principalmente en el contexto de estudios que evalúan el impacto de programas educativos respecto al delirium ${ }^{31-32}$.

Nuestro estudio tiene varias limitaciones. En primer lugar, debido a la metodología empleada obtuvimos respuesta en solo un tercio de las encuestas enviadas. No obstante, considerando el tamaño de nuestra población nacional, la cantidad 
de profesionales que respondieron es comparable en proporción con otros reportes internacionales que han empleado similar metodología ${ }^{19,21}$. En segundo lugar, debido al diseño del estudio, las respuestas entregadas por los profesionales no necesariamente reflejan las prácticas cotidianas de las unidades. Gill y cols. ${ }^{33}$, han demostrado que la percepción respecto a la adherencia, tiene diferencias significativas con la práctica diaria. En tercer lugar, la encuesta aplicada empleó respuestas categóricas, lo que limita el análisis cuantitativo y dificulta las comparaciones con la información internacional. Finalmente, nuestro equipo omitió incorporar alguna estrategia para capturar la información territorial del encuestado, de modo que no podemos estimar la representatividad de cada región en la encuesta nacional.

En conclusión, nuestro estudio entrega información relevante respecto al conocimiento y percepciones de profesionales de la salud en esta área del cuidado del paciente crítico. Existen áreas que impresionan prioritarias de intervenir en futuros proyectos educativos liderados por sociedades científicas y universidades, así como el monitoreo del dolor con instrumentos validados, la aplicación de instrumentos para el diagnóstico del delirium, la implementación de estrategias para la PNFD y la utilización y monitoreo de BNM. Los resultados obtenidos en esta encuesta nacional fueron un valioso recurso para el desarrollo de las Recomendaciones SOCHIMI para la Analgesia, Sedación, Delirium y Bloqueo Neuromuscular en Pacientes Críticos Médico-Quirúrgicos Adultos ${ }^{34}$.

Agradecimientos: Los autores agradecen a la Sociedad Chilena de Medicina Intensiva (SOCHIMI) por su apoyo y a todos los profesionales de salud que respondieron la encuesta.

\section{Referencias}

1. Kress JP, Pohlman AS, O'Connor MF, Hall JB. Daily interruption of sedative infusions in critically ill patients undergoing mechanical ventilation. N Engl J Med. 2000; 342 (20): 1471-77.

2. Brattebø G, Hofoss D, Flaatten H, Muri AK, Gjerde $S$, Plsek PE. Effect of a scoring system and protocol for sedation on duration of patients' need for ventilator support in a surgical intensive care unit. BMJ 2002; 324 (7350): 1386-89.
3. Chanques G, Jaber S, Barbotte E, Violet S, Sebbane M, Perrigault PF, et al. Impact of systematic evaluation of pain and agitation in an intensive care unit. Crit Care Med. 2006; 34 (6): 1691-99.

4. Papazian L, Forel JM, Gacouin A, Penot-Ragon C, Perrin G, Loundou A, et al. Neuromuscular blockers in early acute respiratory distress syndrome. N Engl J Med. 2010; 363 (12): 1107-16.

5. Ely EW, Shintani A, Truman B, Speroff T, Gordon SM, Harrell FE, et al. Delirium as a predictor of mortality in mechanically ventilated patients in the intensive care unit. JAMA 2004; 291 (14): 1753-62.

6. Pisani MA, Kong SY, Kasl SV, Murphy TE, Araujo KL, Van Ness PH. Days of delirium are associated with 1 -year mortality in an older intensive care unit population. Am J Respir Crit Care Med. 2009; 180 (11): $1092-$ 97.

7. Bounds M, Kram S, Speroni KG, Brice K, Luschinski MA, Harte S, et al. Effect of ABCDE Bundle Implementation on Prevalence of Delirium in Intensive Care Unit Patients. Am J Crit Care 2016; 25 (6): 535-44.

8. Girard TD, Kress JP, Fuchs BD, Thomason JW, Schweickert WD, Pun BT, et al. Efficacy and safety of a paired sedation and ventilator weaning protocol for mechanically ventilated patients in intensive care (Awakening and Breathing Controlled trial): a randomised controlled trial. Lancet 2008; 371 (9607): 126-34.

9. Schweickert WD, Pohlman MC, Pohlman AS, Nigos C, Pawlik AJ, Esbrook CL, et al. Early physical and occupational therapy in mechanically ventilated, critically ill patients: a randomised controlled trial. Lancet 2009; 373 (9678): 1874-82.

10. Riker RR, Shehabi Y, Bokesch PM, Ceraso D, Wisemandle W, Koura F, et al. Dexmedetomidine vs midazolam for sedation of critically ill patients: a randomized trial. JAMA 2009; 301 (5): 489-99.

11. Jakob SM, Ruokonen E, Grounds RM, Sarapohja T, Garratt C, Pocock SJ, et al. Dexmedetomidine vs midazolam or propofol for sedation during prolonged mechanical ventilation: two randomized controlled trials. JAMA 2012; 307 (11): 1151-60.

12. Mehta S, Burry L, Fischer S, Martinez-Motta JC, Hallett $\mathrm{D}$, Bowman D, et al. Canadian survey of the use of sedatives, analgesics, and neuromuscular blocking agents in critically ill patients. Crit Care Med. 2006; 34 (2): 374-80.

13. Burry L, Williamson D, Perreault M, Rose L, Cook D, Ferguson N, et al. Analgesic, sedative, antipsychotic, and neuromuscular blocker use in Canadian intensive care units: a prospective, multicentre, observational study. Can J Anaesth 2014; 61 (7): 619-30. 
14. Devlin J, Fong J, Howard E, Skrobik Y, McCoy N, Yasuda C, et al. Assessment of delirium in the intensive care unit: nursing practices and perceptions. Am J Crit Care 2008; 17 (6): 555-65.

15. Xing J, Sun Y, Jie Y, Yuan Z, Liu W. Perceptions, attitudes, and current practices regards delirium in China: A survey of 917 critical care nurses and physicians in China. Medicine (Baltimore) 2017; 96 (39): e8028.

16. Barr J, Fraser GL, Puntillo K, Ely EW, Gélinas C, Dasta $\mathrm{JF}$, et al. Clinical practice guidelines for the management of pain, agitation, and delirium in adult patients in the intensive care unit. Crit Care Med. 2013; 41 (1): 263306.

17. Devlin J, Skrobik Y, Gélinas C, Needham D, Slooter A, Pandharipande $\mathrm{P}$, et al. Clinical practice guidelines for the prevention and management of pain, agitation/ sedation, delirium, immobility, and sleep disruption in adult patients in the ICU. Crit Care Med. 2018; 46 (9): e825-73.

18. Murray MJ, DeBlock H, Erstad B, Gray A, Jacobi J, Jordan C, et al. Clinical practice guidelines for sustained neuromuscular blockade in the adult critically ill patient. Crit Care Med. 2016; 44 (11): 2079-103.

19. Salluh JI, Dal-Pizzol F, Mello PV, Friedman G, Silva E, Teles JM, et al. Delirium recognition and sedation practices in critically ill patients: a survey on the attitudes of 1015 Brazilian critical care physicians. J Crit Care 2009; 24 (4): 556-62.

20. Tobar E, Bugedo G, Andresen M, Aguirre M, Lira MT, Godoy J, et al. Características e impacto de la sedación, la analgesia y el bloqueo neuromuscular en los pacientes críticos que recibieron ventilación mecánica prolongada. Med Intensiva 2009; 33 (7): 311-20.

21. Morandi A, Piva S, Ely EW, Myatra S, Salluh JI, Amare $\mathrm{D}$, et al. Worldwide survey of the "assessing pain, both spontaneous awakening and breathing trials, choice of drugs, delirium monitoring/management, early exercise/ mobility, and family empowerment" (ABCDEF) bundle. Crit Care Med. 2017; 45 (11): e1111-22.

22. Rojas V. Práctica Clínica de Analgesia en las Unidades Intensivas de Santiago. Rev Chil Med Intensiva 2015; 30 (3): 160-61.

23. Tobar E, Lanas MA, Pino S, Aspée P, Rivas S, Prat D, et al. Sedación guiada por protocolo versus manejo convencional en pacientes críticos en ventilación mecánica. Rev Med Chile 2008; 136 (6): 711-18.

24. Augustes R, Ho M. Meta-analysis of randomized controlled trial on daily sedation interruption for critically ill adult patients. Anaesth Intensive Care 2011; 39 (3): 401-9.

25. Mehta S, Burry L, Cook D, Fergusson D, Steinberg M, Granton J, et al. Daily sedation interruption in mechanically ventilated critically ill patients cared for with a sedation protocol: a randomized controlled trial. JAMA 2012; 308 (19): 1985-92.

26. Frazee E, Personett A, Bauer R, Dzierba L, Stollings L, Ryder $\mathrm{P}$, et al Intensive Care Nurses' Knowledge About Use of Neuromuscular Blocking Agents in Patients With Respiratory Failure. Am J Critical Care 2015; 24 (5): 431-39.

27. van den Boogaard M, Slooter AJC, Brüggemann RJM, Schoonhoven L, Beishuizen A, M Vermeijden W, et al. Effect of haloperidol on survival among critically ill adults with a high risk of delirium: the REDUCE randomized clinical trial. JAMA 2018; 319 (7): 680-90.

28. Girard T, Exline M, Carson S, Hough C, Rock P, Gong $\mathrm{M}$, et al. Haloperidol and ziprasidone for treatment of delirium in critical illness. N Engl J Med. 2018; 379: (26): 2506-16.

29. Trogrlić Z, Ista E, Ponssen H, Schoonderbeek F, Schreiner F, Verbrugge J, et al. Attitudes, knowledge and practices concerning delirium: a survey among intensive care unit professionals. Nursing in Critical Care 2016; 22 (3): 133-40.

30. Ozsaban A, Acaroglu R. Delirium assessment in intensive care units: practices and perceptions of Turkish nurses. Nurs Crit Care 2019; 21 (5): 271-8.

31. Dahl L. Delirium Screening Instrument Knowledge and Perception among Cardiovascular Intensive Care Nurses. DNP Projects 2015; 72. https://uknowledge.uky. edu/dnp_etds/72.

32. van den Boogaard $M$, Pickkers $P$, van der Hoeven $H$, Roodbol G, van Achterberg T, Schoonhoven L. Implementation of a delirium assessment tool in the ICU can influence haloperidol use. Critical Care 2009; 13 (4): R131.

33. Gill KV, Voils SA, Chenault GA, Brophy GM. Perceived versus actual sedation practices in adult intensive care unit patients receiving mechanical ventilation. Ann Pharmacother 2012; 46 (10): 1331-9.

34. Tobar E, Rojas V, Álvarez E, Romero CM, Sepúlveda MI, Cariqueo M, et al. Recomendaciones de la Sociedad Chilena de Medicina Intensiva para la analgesia, sedación, delirium y bloqueo neuromuscular en pacientes críticos médico-quirúrgicos adultos. Rev Chil Med Intensiva 2019; 34 (1): 1-29. 